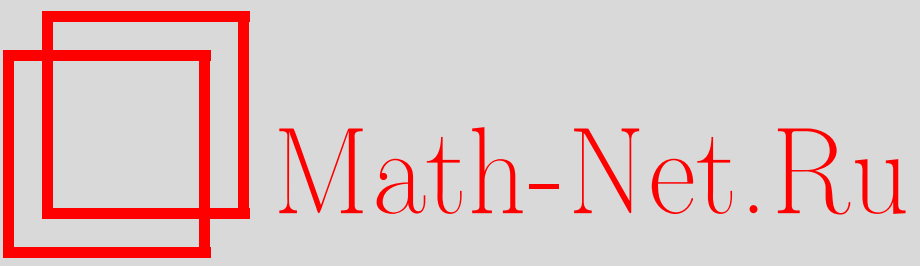

Н. Г. Мощевитин, О пересечении канторовых $\tau$-множеств, УМH, 2002, том 57 , выпуск 1, 159-160

DOI: https://doi.org/10.4213/rm484

Использование Общероссийского математического портала Math-Net.Ru подразумевает, что вы прочитали и согласны с пользовательским соглашением

http://www.mathnet.ru/rus/agreement

Параметры загрузки:

IP: 54.172 .240 .79

26 апреля 2023 г., 14:04:35 


\section{О ПЕРЕСЕЧЕНИИ КАНТОРОВЫХ $\tau$-МНОЖЕСТВ}

\section{Н. Г. МощЕвитин}

\section{1. Результаты о пересечении $\tau$-множеств.}

ОПредЕлЕниЕ. Пусть $\mathscr{I} \subset \mathbb{R}$ - некоторьй отрезок. Множество $F \subset \mathscr{I}$ называется $\tau$-множеством $(\tau>0)$, если оно может быть получено из отрезка $\mathscr{I}$ последовательным выкидыванием счетного (или конечного) набора интервалов $\Delta_{\nu} \subset \mathscr{I}, \nu=1,2, \ldots$, так, что каждьй раз интервал $\Delta_{\nu}$ выкидывается из некоторого отрезка $M_{\nu} \subset \mathscr{I}$, разбивая его таким образом, что

$$
M_{\nu}=N_{1} \sqcup \Delta_{\nu} \sqcup N_{2} \text { и } \min \left\{\left|N_{1}\right|,\left|N_{2}\right|\right\} \geqslant \tau\left|\Delta_{\nu}\right| .
$$

(Через $|\cdot|$ здесь и в дальнейшем обозначается длина отрезка или интервала.)

Совокупность всех выкидьваемых интервалов обозначим $i(F)$, так что $F=\mathscr{I} \backslash \bigcup_{\Delta \in i(F)} \Delta$.

$\tau$-множества с $\tau=1$ использовались Миримановым [1] и Холлом [2]. С произвольным $\tau$ эти множества рассматривались Ньюхаузом [3]-[5] и Вильямсом [6]. Холл [2] доказал, что для 1-множества $F$ сумма $F+F$ является отрезком.

Следующие ниже утверждения обобщают и развивают теорему Холла. Напомним еще одно определение. Два множества назьваются зацепляющимися, если одно из них не лежит целиком в связной компоненте дополнения к другому.

Tеорема А. Пусть $F_{1}$ и $F_{2}$ - соответственно $\tau_{1}-u \tau_{2}$-множества $и \tau_{1} \tau_{2} \geqslant 1$. Если, кроме того, $F_{1}$ и $F_{2}$ зацепляются, то $F_{1} \cap F_{2} \neq \varnothing$.

Теорема А была доказана Ньюхаузом (см. [3]-[5]).

Повторяя рассуждения Холла и Ньюхауза, легко устанавливается следующий чуть более общий факт (см. [7]): если $F_{1}=\mathscr{I}_{1} \backslash \bigcup_{\nu=1}^{\infty} \Delta_{\nu}^{1}, F_{2}=\mathscr{I}_{2} \backslash \bigcup_{\nu=1}^{\infty} \Delta_{\nu}^{2}$ и выполнено $\left|\mathscr{I}_{1}\right| \geqslant\left|\mathscr{I}_{2}\right| \geqslant$ $\max _{\nu \in \mathbb{N}}\left|\Delta_{\nu}^{1}\right|$, то множества $F_{1}+F_{2}$ и $F_{1}-F_{2}$ являются отрезками.

Отметим, что в работе автора [8] и в работах Астелса [7], [9] получены обобщения теоремы Холла-Ньюхауза для сумм $F_{1}+\cdots+F_{r} \tau$-множеств $F_{1}, \ldots, F_{r}$.

В работе Вильямса [6] доказан следующий результат.

Теорема В. 1. Пусть $F_{j}=\mathscr{I}_{j} \backslash \bigcup_{\nu=1}^{\infty} \Delta_{j}^{\nu}, j=1,2$, - два зацепляющихся $\tau$-множества с $\tau>1+\sqrt{2}$. Тогда пересечение $F_{1} \cap F_{2}$ содержит некоторое канторово подмножество $F \subset F_{1} \cap F_{2}$.

2. Для любьх $\tau_{1}, \tau_{2} \in(1,1+\sqrt{2})$ существуют канторовы $\tau_{j}$-множества $F_{j}, j=1,2$, такие, что их пересечение $F_{1} \cap F_{2}$ состоит из одной точки.

3. Пусть $F_{j}=\mathscr{I}_{j} \backslash \bigcup_{\nu=1}^{\infty} \Delta_{j}^{\nu}, j=1,2,-$ два $\tau$-множества с $\tau=1+\sqrt{2}$. Тогда пересечение $F_{1} \cap F_{2}$ содержит бесконечное количество точек.

4. Существуют два $(1+\sqrt{2})$-множсества $F_{1}, F_{2}$ такие, что их пересечение $F_{1} \cap F_{2}$ бесконечно, но счетно.

В этой же работе Вильямса имеются и некоторые другие утверждения.

Основным резултатом настоящей работы является следующее утверждение.

ТеОРема 1. Пусть $F_{j}=\mathscr{I}_{j} \backslash \bigcup_{\nu=1}^{\infty} \Delta_{j}^{\nu}, j=1,2,-$ два зацепляющихся $\tau$-множества с одним и тем же достаточно большим $\tau$. Тогда пересечение $F_{1} \cap F_{2}$ содержит некоторое $\tau_{1}$-множество $F \subset F_{1} \cap F_{2} c \tau_{1}=\sqrt{\tau} / 6+O(1), \tau \rightarrow \infty$.

Отметим, что в отличие от работы [6] мы не стремились получить точные значения констант в теореме 1 , но пытались проследить порядок зависимости величины $\tau_{1}$ от $\tau$ с ростом параметра $\tau$.

2. О доказательстве теоремы 1. Доказательство теоремы 1 содержит довольно громоздкие выкладки. Здесь мы приведем лишь его идею.

Через $U_{\delta}(A)$ будем обозначать $\delta$-окрестность множества $A$. Для интервалов $\Delta^{\prime}, \Delta^{\prime \prime} \in i\left(F_{1}\right) \cup$ $i\left(F_{2}\right)$ будем писать $\Delta^{\prime} \| \Delta^{\prime \prime}$, если $\Delta^{\prime}$ и $\Delta^{\prime \prime}$ принадлежат одному и тому же $i\left(F_{j}\right)$. Если же $\Delta^{\prime} \in$ $i\left(F_{j}\right), \Delta^{\prime \prime} \in i\left(F_{k}\right)$ и $j \neq k$, то будем писать $\Delta^{\prime} \perp \Delta^{\prime \prime}$.

Работа выполнена при финансовой поддержке Российского фонда фундаментальных исследований (гранты № 99-01-00357, 00-15-96109) и фонда INTAS. 
Для $\Delta \in i\left(F_{1}\right) \cup i\left(F_{2}\right)$ определим семейство

$$
\begin{gathered}
\mathscr{G}(\Delta)=\left\{\Delta^{\prime} \in i\left(F_{1}\right) \cup i\left(F_{2}\right): \exists \Delta^{0}=\Delta, \Delta^{1}, \ldots, \Delta^{\nu}=\Delta^{\prime}\right. \text { такие, что } \\
\left.\left|\Delta^{j}\right| \geqslant\left|\Delta^{j+1}\right|, \Delta^{j} \cap U_{\sqrt{\tau}\left|\Delta^{j+1}\right|}\left(\Delta^{j+1}\right) \neq \varnothing, j=0,1, \ldots, \nu-1\right\}, \\
\sigma(\Delta)=\operatorname{conv}\left[\bigcup_{\Delta^{\prime} \in \mathscr{G}(\Delta)} \Delta^{\prime}\right] .
\end{gathered}
$$

Заметим, что если $\tau>1$, то в каждой цепи интервалов $\Delta^{j}$ из определения совокупности $\mathscr{G}$ выполнено $\Delta^{j} \perp \Delta^{j+1}, j=0,1, \ldots, \nu-1$.

Теорема 1 доказывается в два этапа.

На первом этапе доказьвается, что при условии $\tau \geqslant 9$ множество $F_{1} \cap F_{2}$ содержит множество $F=\mathscr{I} \backslash \bigcup_{k} \sigma_{k} \subset F_{1} \cap F_{2}$ (где $\sigma_{k}=\sigma\left(\Delta_{j_{k}}^{\nu_{k}}\right)$ ), которое может быть получено последовательным вькидыванием из отрезка $\mathscr{I}_{1} \cap \mathscr{I}_{2}$ конечного или счетного набора непересекающихся интервалов $\sigma_{k}$ (выбираем максимальное по длине $\Delta \in i\left(F_{1}\right) \cup i\left(F_{2}\right)$ и выкидьваем $\sigma_{1}=\sigma(\Delta)$, затем среди всех оставшихся не выкинутых вместе с $\sigma(\Delta)$ интервалов выбираем максимальньй по длине $\Delta^{\prime}$ и вькидываем $\sigma_{2}=\sigma\left(\Delta^{\prime}\right)$ и так далее). То, что при такой процедуре вькидывания $\sigma_{k}$ и $\sigma_{k+1}$ действительно на пересекаются, является основным вспомогательным утверждением, которое надо доказать на первом этапе. Доказательство проводится разбором двух случаев: $\Delta_{j_{k}}^{\nu_{k}} \| \Delta_{j_{k+1}}^{\nu_{k+1}}$ и $\Delta_{j_{k}}^{\nu_{k}} \perp \Delta_{j_{k+1}}^{\nu_{k+1}}$.

На втором этапе доказьвается, что при достаточно большом $\tau F$ будет $\tau_{1}$-множеством. При этом случаи $\Delta_{j_{k}}^{\nu_{k}} \| \Delta_{j_{k+1}}^{\nu_{k+1}}$ и $\Delta_{j_{k}}^{\nu_{k}} \perp \Delta_{j_{k+1}}^{\nu_{k+1}}$ тоже разбираются по-разному.

3. Замечания. То, что некоторое множество $A \subset[0,1]$ является $\tau$-множеством с некоторьгм положительным $\tau$, по сути своей есть очень сильное условие. Приведем два примера.

1. $N_{q}$, где $q$ есть целое, большее $1,-$ множество всех чисел $\alpha \in \mathbb{R}$, для которых последовательность дробных долей $\left\{\alpha q^{l}\right\}, l=1,2,3, \ldots$, всюду плотна в отрезке $[0,1]$.

2. $K$-множество всех чисел отрезка $[0,1]$, разложения которых в регулярную цепную дробь имеет последовательность неограниченных неполных частных.

$K$ и каждое $N_{q}$ являются множествами полной меры, но очевидньм следствием теоремы А Ньюхауза является то, что в $N_{q}$ и $K$ нет ни одного $\tau$-подмножества. В [10] дается такое объяснение этого факта: множество $\mathfrak{A} \subset 2^{[0,1]}$ всех $\tau$-множеств из отрезка $[0,1]$ в экспоненциальном пространстве $\exp ([0,1])$ с метрикой Хаусдорфа является множеством первой категории Бэра, т.е. распадается в счетное объединение нигде не плотных множеств.

(Пользуясь случаем, отметим, что пересечение $\bigcap_{q \geqslant 2}\left([0,1] \backslash N_{q}\right)$ непусто: как показал Шмидт [11], это следует из $\alpha$-вьшгрьшности каждого [0, 1$] \backslash N_{q} ;$ с другой стороны, явные примеры чисел, не являющихся нормальньци ни по какому основанию $q$, построенные в виде специальных $p я д о в$, имеются у Коробова [12].)

\section{СПИСОК ЛИТЕРАТУРЫ}

[1] D. Mirimanoff // Fund. Math. 1923. V. 4. P. 118-121. [2] M. Jr. Hall // Ann. of Math. (2). 1948. V. 48. P. 966-993. [3] S. Newhouse // Proc. Sympos. Pure Math. 1970. V. 14. P. 191-202. [4] S. Newhouse // Topology. 1974. V. 13. P. 9-18. [5] S. Newhouse // Inst. Hautes Études Sci. Publ. Math. 1979. V. 50. P. 101-152. [6] R. F. Williams // Contemp. Math. 1991. V. 117. P. 163-175. [7] S. Astels // Electron. Res. Announc. Amer. Math. Soc. 1999. V. 5. № 15. Р. 108-111. [8] Н. Г. Мощевитин // Матем. заметки. 2000. Т. 68. № 6. С. 948-950. [9] S. Astels // Trans. Amer. Math. Soc. 2000. V. 352. №1. P. 133-170. [10] Н. Г. Мощевитин // УМH. 1999. T. 54. № 3. C. 169-170. [11] W. M. Schmidt // Trans. Amer. Math. Soc. 1966. V. 123. Р. 178-199. [12] А. Н. Коробов. Диофантовы приближения. М.: Центр прикладных исследований при механико-математическом факулттете МГУ, 1996.

Московский государственньй

Принято редколлегией 